Global Hölder estimates for hypoelliptic operators with drift on homogeneous groups

Yuexia Hou, Xiaojing Feng, and Xuewei Cui 


\title{
GLOBAL HÖLDER ESTIMATES FOR HYPOELLIPTIC OPERATORS WITH DRIFT ON HOMOGENEOUS GROUPS
}

\author{
YUEXIA HOU, XIAOJING FENG, AND XUEWEI CUI
}

Received 19 December, 2011

\begin{abstract}
Let $X_{0}, X_{1}, \ldots, X_{q}$ be left invariant real vector fields on the homogeneous group $G$, satisfying Hörmander's condition on $\mathbb{R}^{N}$. Assume that $X_{1}, \ldots, X_{q}$ are homogeneous of degree one and $X_{0}$ is homogeneous of degree two. In this paper we consider the following hypoelliptic operator with drift

$$
L=\sum_{i, j=1}^{q} a_{i j} X_{i} X_{j}+a_{0} X_{0},
$$

where $\left(a_{i j}\right)$ is a $q \times q$ positive constant matrix and $a_{0} \neq 0$, and obtain Global Hölder estimates for $L$ on $G$ by establishing several estimates of singular integrals.
\end{abstract}

2000 Mathematics Subject Classification: 20D15; 35B65; 35C15

Keywords: homogeneous group, singular integral, global Hölder estimate

\section{INTRODUCTION}

Let $G$ be a homogeneous group and $X_{0}, X_{1}, \ldots, X_{q}$ be left invariant real vector fields on $\mathbb{R}^{N}(q<N)$. Assume that $X_{1}, \ldots, X_{q}$ are homogeneous of degree one and $X_{0}$ is homogeneous of degree two, satisfying Hörmander's condition

$$
\operatorname{rank} \mathscr{L}\left(X_{0}, X_{1}, \ldots, X_{q}\right)(x)=N, x \in \mathbb{R}^{N},
$$

where $\mathscr{L}\left(X_{0}, X_{1}, \ldots, X_{q}\right)$ denotes the Lie algebra generated by $X_{0}, X_{1}, \ldots, X_{q}$. In this paper we are interested in the following hypoelliptic operator with drift

$$
L=\sum_{i, j=1}^{q} a_{i j} X_{i} X_{j}+a_{0} X_{0},
$$

This work was supported by the National Natural Science Foundation of China (Grant Nos. 10871157 and 11001221); Specialized Research Found for the Doctoral Program of Higher Education (No. 200806990032) and the Mathematical Tianyuan Foundation of China (Grant No. 11126027). 
where $a_{0} \neq 0,\left(a_{i j}\right)_{i, j=1}^{q}$ is a constant matrix satisfying

$$
\mu^{-1}|\xi|^{2} \leq \sum_{i, j=1}^{q} a_{i j} \xi_{i} \xi_{j} \leq \mu|\xi|^{2}, \xi \in R^{q},
$$

for a constant $\mu>0$.

Many authors paid attention to the hypoelliptic operator. The outstanding result in [8] points out that Hörmander's condition implies (actually, is equivalent to) the hypoellipticity of $L$ in (1.1). The existence of fundamental solutions for homogeneous hypoelliptic operators on nilpotent Lie groups was investigated by Folland in [6]. Bramanti and Brandolini in [2] proved the uniqueness of homogeneous fundamental solutions for $L$. Let us note that $L$ includes the classic Laplace operator and parabolic operator on Euclidean spaces. Another special case of $L$ is

$$
L_{1}=\sum_{i, j=1}^{q} a_{i j} \partial_{x_{i} x_{j}}^{2}+\sum_{i, j=1}^{n} b_{i j} x_{i} \partial_{x_{j}}-\partial_{t},
$$

where $(x, t) \in \mathbb{R}^{n+1}, X_{0}=\sum_{i, j=1}^{n} b_{i j} x_{i} \partial_{x_{j}}-\partial_{t}$ and $X_{i}=\partial_{x_{i}}, i=1,2, \ldots, q,\left(a_{i j}\right)_{i, j=1}^{q}$ is a positive matrix in $R^{q}$, $\left(b_{i j}\right)$ is a constant matrix with a suitable upper triangular structure. Note that $L_{1}$ belongs to a class of Kolmogorov-Fokker-Planck ultraparabolic operators. The operator $L_{1}$ appears in many research fields, for instance, in stochastic processes and kinetic models (see [3-5]), and in mathematical finance theory (see [1,12]). After the previous study on $L_{1}$ in $[9,10]$, the authors of $[7,11,13]$ established an invariant Harnack inequality for the non-negative solution of $L_{1} u=0$ by applying the mean value formula. With the theory of singular integral, Polidoro and Ragusa in [14] concluded some Morrey-type imbedding results and gave a local Hölder continuity of the solution.

The aim of the paper is to prove global Hölder estimates on the homogeneous group $G$ for $L$ by applying the properties of the fundamental solution for $L$ and several estimates of singular integrals on the homogeneous space. The method here is inspired by that used in [14]. Our results reflect the relations between the Morrey norms of $L u$ and Hölder exponents for $u$ and $X_{i} u, i=1,2, \ldots, q$. In order to state our main results, we first introduce the definition of Morrey space.

Definition 1. For $p \in(1, \infty), \lambda \in[0, Q)$, the Morrey space on homogeneous group $G$ is defined by

$$
L^{p, \lambda}(G)=\left\{g \in L_{l o c}^{p}(G):\|g\|_{L^{p, \lambda}(G)}<\infty\right\},
$$

where

$$
\|g\|_{L^{p, \lambda}(G)}=\left(\sup _{r>0, x \in G} \int_{B_{r}(x)} \frac{1}{r^{\lambda}}|g(y)|^{p} d y\right)^{1 / p},
$$

$B_{r}(x)$ and $Q$ will be given in (2.1) and (2.2), respectively. Here $L^{p, 0}(G)=L^{p}(G)$. 
The main results of this paper are as follows. For the case $\lambda \neq 0$, we have

Theorem 1. (1) If $1<p<\frac{Q}{2}, Q-2 p<\lambda<Q-p$, then there exists a positive constant $c=c(p, \lambda)$ such that for every $u \in C_{0}^{\infty}(G)$ and any $x, z \in$ $G, x \neq z$,

$$
\frac{|u(x)-u(z)|}{\left\|z^{-1} \circ x\right\|^{\theta}} \leq c\|L u\|_{L^{p, \lambda}(G)},
$$

where $\theta=\frac{2 p+\lambda-Q}{p}$;

(2) If $1<p<\frac{Q}{2}, Q-p<\lambda<Q$, then there exists a positive constant $c=$ $c(p, \lambda)$ such that for every $u \in C_{0}^{\infty}(G)$ and any $x, z \in G, x \neq z$,

$$
\frac{\left|X_{i} u(x)-X_{i} u(z)\right|}{\left\|z^{-1} \circ x\right\|^{\theta}} \leq c\|L u\|_{L^{p, \lambda}(G)},
$$

where $i=1, \cdots, q$ and $\theta=\frac{p+\lambda-Q}{p}$.

For $\lambda=0$, we have the following results, which restores the known result previously proved in [1].

Remark 1. (1) Assume $\frac{Q}{2}<p<Q$. Then there exists a positive constant $c=c(p)$ such that for every $u \in C_{0}^{\infty}(G)$ and any $x, z \in G, x \neq z$,

$$
\frac{|u(x)-u(z)|}{\left\|z^{-1} \circ x\right\|^{\theta}} \leq c\|L u\|_{L^{p}(G)},
$$

where $\theta=\frac{2 p-Q}{p}$;

(2) Assume $p>Q$. Then there exists a positive constant $c=c(p)$ such that for every $u \in C_{0}^{\infty}(G)$ and any $x, z \in G, x \neq z$,

$$
\frac{\left|X_{i} u(x)-X_{i} u(z)\right|}{\left\|z^{-1} \circ x\right\|^{\theta}} \leq c\|L u\|_{L^{p}(G)},
$$

where $i=1, \cdots, q$ and $\theta=\frac{p-Q}{p}$.

The plan of the paper is as follows: in Section 2 we introduce some knowledge of homogeneous group and related lemmas. Estimates of two integral operators are proved. Section 3 is devoted to the proof of the main result.

\section{PReliminary}

Given a pair of mappings:

$$
[(x, y) \mapsto x \circ y]: \mathbb{R}^{N} \times \mathbb{R}^{N} \mapsto \mathbb{R}^{N} ;\left[x \mapsto x^{-1}\right]: \mathbb{R}^{N} \mapsto \mathbb{R}^{N},
$$


which are smooth, it follows that $\mathbb{R}^{N}$ with these mappings forms a group, and the identity is the origin. If there exist $0<\omega_{1} \leq \omega_{2} \leq \ldots \leq \omega_{N}$, such that the dilations

$$
D(\lambda):\left(x_{1}, \ldots, x_{N}\right) \mapsto\left(\lambda^{\omega_{1}} x_{1}, \ldots, \lambda^{\omega_{N}} x_{N}\right), \lambda>0,
$$

are group automorphisms, then the space $\mathbb{R}^{N}$ with this structure is called a homogeneous group and denoted by $G$.

Definition 2. We define a homogeneous norm $\|\cdot\|$ in $G$ by the following way: if for any $x \in G, x \neq 0$, it holds

$$
\|x\|=\rho \quad \Leftrightarrow \quad|D(1 / \rho) x|=1,
$$

where $|\cdot|$ denotes the Euclidean norm; also, let $\|0\|=0$.

It is not difficult to derive that the homogeneous norm satisfies

(1) $\|D(\lambda) x\|=\lambda\|x\|$ for every $x \in G, \lambda>0$;

(2) there exists $c(G) \geq 1$, such that for every $x, y \in G$,

$$
\left\|x^{-1}\right\| \leq c\|x\| \text { and }\|x \circ y\| \leq c(\|x\|+\|y\|) .
$$

In view of the above properties, it is natural to define the quasidistance $d$ :

$$
d(x, y)=\left\|y^{-1} \circ x\right\| .
$$

The ball with respect to $d$ is denoted by

$$
B(x, r) \equiv B_{r}(x)=\{y \in G: d(x, y)<r\} .
$$

Note $B(0, r)=D(r) B(0,1)$, therefore

$$
|B(x, r)|=r^{Q}|B(0,1)|, x \in G, r>0,
$$

where

$$
Q=\omega_{1}+\ldots+\omega_{N}
$$

We will call that $Q$ is the homogeneous dimension of $G$. In general, $Q \geq 3$.

Definition 3. A differential operators $Y$ on $G$ is said homogeneous of degree $\beta(\beta>0)$, if for every test function $\varphi$,

$$
Y(\varphi(D(\lambda) x))=\lambda^{\beta}(Y \varphi)(D(\lambda) x), \lambda>0, x \in G ;
$$

A function $f$ is called homogeneous of degree $\alpha$, if

$$
f((D(\lambda) x))=\lambda^{\alpha} f(x), \lambda>0, x \in G .
$$

Remark 2. Clearly, if $Y$ is a differential operators of homogeneous of degree $\beta$ and $f$ is a function of homogeneous of degree $\alpha$, then $Y f$ is homogeneous of degree $\alpha-\beta$.

Lemma 1. ([2]) The operator $L$ possesses a unique fundamental solution $\Gamma(\cdot)$, such that for every test function $u \in C_{0}^{\infty}(G)$ and every $x \in G$, it holds

(1) $\Gamma(\cdot) \in C^{\infty}(G \backslash\{0\})$; 
(2) $\Gamma(\cdot)$ is homogeneous of degree $2-Q$;

(3) $u(x)=(L u * \Gamma)(x)=\int_{\mathbb{R}^{N}} \Gamma\left(y^{-1} \circ x\right) L u(y) d y$;

(4) $X_{i} u(x)=\int_{R^{N}} X_{i} \Gamma\left(y^{-1} \circ x\right) L u(y) d y$.

Remark 3. If we set $\Gamma_{i}=X_{i} \Gamma, i=1, \cdots, q$, then it is obvious from Remark 2 that $\Gamma_{i}(\cdot)$ is homogeneous of degree $1-Q$.

Proposition 1. ([2]) Let $f \in C^{1}\left(R^{N} \backslash 0\right)$ is a homogeneous function of degree $\lambda<1$. Then there exist two constants $c=c(G, f)>0$ and $M=M(G)>1$, such that for any $x, y$ satisfying $\|x\| \geq M\|y\|$,

$$
|f(x \circ y)-f(x)|+|f(y \circ x)-f(x)| \leq c\|y\|\|x\|^{\lambda-1},
$$

where $c=c(G, f) \sup _{z \in \Sigma_{N}}|\nabla f(z)|, \Sigma_{N}$ is the unit sphere of $\mathbb{R}^{N}$.

From Proposition 1, it follows

Lemma 2. If $K \in C^{1}(G \backslash\{0\})$ is a homogeneous function of degree $\alpha<1$ with respect to the group $(D(\lambda))_{\lambda>0}$, then there exist two constants $c>0$ and $M>1$, such that if $\|x\| \geq M\left\|x^{-1} \circ z\right\|$, then

$$
|K(z)-K(x)| \leq \frac{c\left\|x^{-1} \circ z\right\|}{\|x\|^{1-\alpha}} .
$$

By Lemma 1 and Lemma 2, we have immediately

Lemma 3. For every $x, y, z \in G$, it holds

(1) there exists a constant $c>0$, such that

$$
\begin{aligned}
& \Gamma\left(y^{-1} \circ x\right) \leq \frac{c}{\left\|y^{-1} \circ x\right\|^{Q-2}} ; \\
& \Gamma_{i}\left(y^{-1} \circ x\right) \leq \frac{c}{\left\|y^{-1} \circ x\right\|^{Q-1}} .
\end{aligned}
$$

(2) there exist two constants $c>0$ and $M>1$, such that if $\left\|y^{-1} \circ x\right\| \geq M\left\|x^{-1} \circ z\right\|$, then

$$
\begin{gathered}
\left|\Gamma\left(y^{-1} \circ x\right)-\Gamma\left(y^{-1} \circ z\right)\right| \leq \frac{c\left\|x^{-1} \circ z\right\|}{\left\|y^{-1} \circ x\right\|^{Q-1}} ; \\
\left|\Gamma_{i}\left(y^{-1} \circ x\right)-\Gamma_{i}\left(y^{-1} \circ z\right)\right| \leq \frac{c\left\|x^{-1} \circ z\right\|}{\left\|y^{-1} \circ x\right\|^{Q}} .
\end{gathered}
$$

Now let us introduce two integral operators. For $p \in(1, \infty)$ and $\lambda \in[0, Q)$, fixed $z \in G$ and $\sigma>0$, we define for every $g \in L^{p, \lambda}(G)$ that

$$
T_{\alpha} g(x)=\int_{\left\|y^{-1} \circ x\right\| \geq \sigma\left\|z^{-1} \circ x\right\|} \frac{g(y)}{\left\|y^{-1} \circ x\right\|^{Q-\alpha}} d y, \alpha \in[0, Q) ;
$$




$$
T^{\beta} g(x)=\int_{\left\|y^{-1} \circ x\right\|<\sigma\left\|z^{-1} \circ x\right\|} \frac{g(y)}{\left\|y^{-1} \circ x\right\|^{Q-\beta}} d y, \beta \in(0, Q) .
$$

Lemma 4. If $\lambda+p \alpha<Q$, then there exists $c=c(p, \lambda, \alpha, \sigma)>0$, such that

$$
\left|T_{\alpha} g(x)\right| \leq c\|g\|_{L^{p, \lambda}(G}\left\|z^{-1} \circ x\right\|^{\frac{p \alpha+\lambda-Q}{p}} ;
$$

if $\lambda+p \beta>Q$, then there exists $c=c(p, \lambda, \beta, \sigma)>0$, such that

$$
\left|T^{\beta} g(x)\right| \leq c\|g\|_{L^{p, \lambda}(G)}\left\|z^{-1} \circ x\right\|^{\frac{p \beta+\lambda-Q}{p}} .
$$

Proof. We follow the idea of Polidoro and Ragusa in [14]. If $\lambda+p \alpha<Q$, then it obtains by decomposing the domain of integration and applying the Hölder inequality that

$$
\begin{aligned}
& \left|T_{\alpha} g(x)\right| \leq \sum_{k=1}^{\infty} \int_{2^{k-1} \sigma\left\|z^{-1} \circ x\right\| \leq\left\|y^{-1} \circ x\right\|<2^{k} \sigma\left\|z^{-1} \circ x\right\|} \frac{g(y)}{\left\|y^{-1} \circ x\right\|^{Q-\alpha}} d y \\
& \leq \sum_{k=1}^{\infty}\left(\frac{1}{2^{k-1} \sigma\left\|z^{-1} \circ x\right\|}\right)^{Q-\alpha} \int_{B_{2^{k} \sigma\left\|z^{-1} \circ x\right\|}(x)}|g(y)| d y \\
& \leq \sum_{k=1}^{\infty}\left(\frac{1}{2^{k-1} \sigma\left\|z^{-1} \circ x\right\|}\right)^{Q-\alpha}\left(\int_{B_{2^{k} \sigma\left\|z^{-1} \circ x\right\|}(x)}|g(y)|^{p} d y\right)^{\frac{1}{p}} \\
& \left|B_{2^{k} \sigma\left\|z^{-1} \circ x\right\|}(x)\right|^{\frac{p-1}{p}} \\
& \leq c \sum_{k=1}^{\infty}\left(\frac{1}{2^{k-1} \sigma\left\|z^{-1} \circ x\right\|}\right)^{Q-\alpha}\left(2^{k} \sigma\left\|z^{-1} \circ x\right\|\right)^{\frac{\lambda}{p}}\|g\|_{L^{p, \lambda}(G)} \\
& \left(2^{k} \sigma\left\|z^{-1} \circ x\right\|\right)^{\frac{(p-1) Q}{p}} \\
& \leq c\|g\|_{L^{p, \lambda}(G)}\left\|z^{-1} \circ x\right\|^{\frac{p \alpha+\lambda-Q}{p}} \sum_{k=1}^{\infty}\left(2^{\frac{p \alpha+\lambda-Q}{p}}\right)^{k} .
\end{aligned}
$$

So (2.3) is proved, since the above series is convergent.

Similarly, if $\lambda+p \beta>Q$, then

$$
\begin{aligned}
& \left|T^{\beta} g(x)\right| \leq \sum_{k=1}^{\infty} \int_{2^{-k} \sigma\left\|z^{-1} \circ x\right\| \leq\left\|y^{-1} \circ x\right\|<2^{1-k} \sigma\left\|z^{-1} \circ x\right\|} \frac{g(y)}{\left\|y^{-1} \circ x\right\|^{Q-\beta}} d y \\
& \leq \sum_{k=1}^{\infty}\left(\frac{1}{2^{-k} \sigma\left\|z^{-1} \circ x\right\|}\right)^{Q-\beta} \int_{B_{2^{1-k} \sigma\left\|z^{-1} \circ x\right\|(x)}}|g(y)| d y
\end{aligned}
$$




$$
\begin{aligned}
\leq & \sum_{k=1}^{\infty}\left(\frac{1}{2^{-k} \sigma\left\|z^{-1} \circ x\right\|}\right)^{Q-\beta}\left(\int_{B_{2^{1-k} \sigma \| z^{-1} \circ x}(x)}|g(y)|^{p} d y\right)^{\frac{1}{p}} \\
& \left|B_{2^{1-k} \sigma\left\|z^{-1} \circ x\right\|}(x)\right|^{\frac{p-1}{p}} \\
\leq & c \sum_{k=1}^{\infty}\left(\frac{1}{2^{-k} \sigma\left\|z^{-1} \circ x\right\|}\right)^{Q-\beta}\left(2^{1-k} \sigma\left\|z^{-1} \circ x\right\|\right)^{\frac{\lambda}{p}}\|g\|_{L^{p, \lambda}(G)} \\
& \left(2^{1-k} \sigma\left\|z^{-1} \circ x\right\|\right)^{\frac{(p-1) Q}{p}} \\
\leq & c\|g\|_{L^{p, \lambda}(G)}\left\|z^{-1} \circ x\right\|^{\frac{p \beta+\lambda-Q}{p}} \sum_{k=1}^{\infty}\left(2^{\frac{Q-p \beta-\lambda}{p}}\right)^{k} .
\end{aligned}
$$

This proves (2.4).

Remark 4. In particular, when $\lambda=0$, we see that if $p \alpha<Q$, then there exists a constant $c=c(p, \alpha, \sigma)>0$, such that

$$
\left|T_{\alpha} g(x)\right| \leq c\|g\|_{L^{p}(G)}\left\|z^{-1} \circ x\right\|^{\frac{p \alpha-Q}{p}} ;
$$

if $p \beta>Q$, then there exists a constant $c=c(p, \beta, \sigma)>0$, such that

$$
\left|T^{\beta} g(x)\right| \leq c\|g\|_{L^{p}(G)}\left\|z^{-1} \circ x\right\|^{\frac{p \beta-Q}{p}} .
$$

\section{Proofs of The MAin Results}

Proof of Theorem 1. (1) With the help of (3) in Lemma 1 and Lemma 3, we know that there exist constants $c>0$ and $M>1$ such that

$$
\begin{aligned}
|u(x)-u(z)|= & \left|\int_{\mathbb{R}^{N}} \Gamma\left(y^{-1} \circ x\right)-\Gamma\left(y^{-1} \circ z\right) L u(y) d y\right| \\
\leq & \int_{\mathbb{R}^{N}}\left|\Gamma\left(y^{-1} \circ x\right)-\Gamma\left(y^{-1} \circ z\right)\right||L u(y)| d y \\
\leq & \int_{\left\|y^{-1} \circ x\right\| \geq M\left\|x^{-1} \circ z\right\|}\left|\Gamma\left(y^{-1} \circ x\right)-\Gamma\left(y^{-1} \circ z\right)\right||L u(y)| d y \\
& +\int_{\left\|y^{-1} \circ x\right\|<M\left\|x^{-1} \circ z\right\|}\left|\Gamma\left(y^{-1} \circ x\right)-\Gamma\left(y^{-1} \circ z\right)\right||L u(y)| d y \\
\leq & \int_{\left\|y^{-1} \circ x\right\| \geq M\left\|x^{-1} \circ z\right\|}\left|\Gamma\left(y^{-1} \circ x\right)-\Gamma\left(y^{-1} \circ z\right)\right||L u(y)| d y \\
& +\int_{\left\|y^{-1} \circ x\right\|<M\left\|x^{-1} \circ z\right\|}\left|\Gamma\left(y^{-1} \circ x\right)\right||L u(y)| d y
\end{aligned}
$$




$$
\begin{aligned}
& +\int_{\left\|y^{-1} \circ x\right\|<M\left\|x^{-1} \circ z\right\|}\left|\Gamma\left(y^{-1} \circ z\right)\right||L u(y)| d y \\
\leq & \int_{\left\|y^{-1} \circ x\right\| \geq M\left\|x^{-1} \circ z\right\|} \frac{c\left\|x^{-1} \circ z\right\|}{\left\|y^{-1} \circ x\right\|^{Q-1}}|L u(y)| d y \\
& +\int_{\left\|y^{-1} \circ x\right\|<M\left\|x^{-1} \circ z\right\| \|} \frac{c}{\left\|y^{-1} \circ x\right\|^{Q-2}}|L u(y)| d y \\
& +\int_{\left\|y^{-1} \circ x\right\|<M\left\|x^{-1} \circ z\right\|} \frac{c}{\left\|y^{-1} \circ z\right\|^{Q-2}}|L u(y)| d y .
\end{aligned}
$$

Noting that if $\left\|y^{-1} \circ x\right\| \geq M\left\|x^{-1} \circ z\right\|$, then

$$
\left\|y^{-1} \circ x\right\| \geq M\left\|x^{-1} \circ z\right\| \geq \frac{M}{c}\left\|z^{-1} \circ x\right\| ;
$$

if $\left\|y^{-1} \circ x\right\|<M\left\|x^{-1} \circ z\right\|$, then

$$
\left\|y^{-1} \circ x\right\|<M c\left\|z^{-1} \circ x\right\|
$$

and

$$
\begin{aligned}
\left\|y^{-1} \circ z\right\| & \leq c\left(\left\|y^{-1} \circ x\right\|+\left\|x^{-1} \circ z\right\|\right)<c\left(M\left\|x^{-1} \circ z\right\|+\left\|x^{-1} \circ z\right\|\right) \\
& =c(1+M)\left\|x^{-1} \circ z\right\|,
\end{aligned}
$$

it follows

$$
\begin{aligned}
|u(x)-u(z)| \leq & \int_{\left\|y^{-1} \circ x\right\| \geq \frac{M}{c}\left\|z^{-1} \circ x\right\|} \frac{c\left\|x^{-1} \circ z\right\|}{\left\|y^{-1} \circ x\right\|^{Q-1}}|L u(y)| d y \\
& +\int_{\left\|y^{-1} \circ x\right\|<M c\left\|z^{-1} \circ x\right\|} \frac{c}{\left\|y^{-1} \circ x\right\|^{Q-2}}|L u(y)| d y \\
& +\int_{\left\|y^{-1} \circ z\right\|<c(1+M)\left\|x^{-1} \circ z\right\|\left\|y^{-1} \circ z\right\|^{Q-2}}|L u(y)| d y \\
\doteq & I_{1}+I_{2}+I_{3} .
\end{aligned}
$$

Applying Lemma 4 ( $\alpha=1$ and $\sigma=\frac{M}{c}$ ) and noting $\lambda+p<Q$, there exists a constant $c=c(p, \lambda, \sigma)>0$ such that

$I_{1} \leq c\|L u\|_{L^{p, \lambda}(G)}\left\|z^{-1} \circ x\right\|\left\|z^{-1} \circ x\right\|^{\frac{p+\lambda-Q}{p}}=c\|L u\|_{L^{p, \lambda}(G)}\left\|z^{-1} \circ x\right\|^{\frac{2 p+\lambda-Q}{p}} ;$

from Lemma 4 ( $\beta=2$ and $\sigma=M c ; \beta=2$ and $\sigma=c(1+M)$, respectively) and $\lambda+2 p>Q$, it follows

$$
I_{2} \leq c\|L u\|_{L^{p, \lambda}(G)}\left\|z^{-1} \circ x\right\|^{\frac{2 p+\lambda-Q}{p}}
$$


and

$$
I_{3} \leq c\|L u\|_{L^{p, \lambda}(G)}\left\|z^{-1} \circ x\right\|^{\frac{2 p+\lambda-Q}{p}} .
$$

In conclusion, we deduce (1.3).

(2) We know from (4) in Lemma 1 and Lemma 3 that there exist two constants $c>0$ and $M>1$ such that

$$
\begin{aligned}
\left|X_{i} u(x)-X_{i} u(z)\right|= & \left|\int_{\mathbb{R}^{N}} \Gamma_{i}\left(y^{-1} \circ x\right)-\Gamma_{i}\left(y^{-1} \circ z\right) L u(y) d y\right| \\
\leq & \int_{\mathbb{R}^{N}}\left|\Gamma_{i}\left(y^{-1} \circ x\right)-\Gamma_{i}\left(y^{-1} \circ z\right)\right||L u(y)| d y \\
\leq & \int_{\left\|y^{-1} \circ x\right\| \geq M\left\|x^{-1} \circ z\right\|}\left|\Gamma_{i}\left(y^{-1} \circ x\right)-\Gamma_{i}\left(y^{-1} \circ z\right)\right||L u(y)| d y \\
& +\int_{\left\|y^{-1} \circ x\right\|<M\left\|x^{-1} \circ z\right\|}\left|\Gamma_{i}\left(y^{-1} \circ x\right)-\Gamma_{i}\left(y^{-1} \circ z\right)\right||L u(y)| d y \\
\leq & \int_{\left\|y^{-1} \circ x\right\| \geq M\left\|x^{-1} \circ z\right\|}\left|\Gamma_{i}\left(y^{-1} \circ x\right)-\Gamma_{i}\left(y^{-1} \circ z\right)\right||L u(y)| d y \\
& +\int_{\left\|y^{-1} \circ x\right\|<M\left\|x^{-1} \circ z\right\|}\left|\Gamma_{i}\left(y^{-1} \circ x\right)\right||L u(y)| d y \\
& +\int_{\left\|y^{-1} \circ x\right\|<M\left\|x^{-1} \circ z\right\|}\left|\Gamma_{i}\left(y^{-1} \circ z\right)\right||L u(y)| d y \\
\leq & \int_{\left\|y^{-1} \circ x\right\| \geq M\left\|x^{-1} \circ z\right\|} \frac{c\left\|x^{-1} \circ z\right\|}{\left\|y^{-1} \circ x\right\|^{Q}|L u(y)| d y} \\
& +\int_{\left\|y^{-1} \circ x\right\|<M\left\|x^{-1} \circ z\right\|} \frac{c}{\left\|y^{-1} \circ x\right\|^{Q-1}|L u(y)| d y} \\
& +\int_{\left\|y^{-1} \circ x\right\|<M\left\|x^{-1} \circ z\right\| \|} \frac{c}{\left\|y^{-1} \circ z\right\|^{Q-1}}|L u(y)| d y .
\end{aligned}
$$

Let us remark that if $\left\|y^{-1} \circ x\right\| \geq M\left\|x^{-1} \circ z\right\|$, then

$$
\left\|y^{-1} \circ x\right\| \geq \frac{M}{c}\left\|z^{-1} \circ x\right\| ;
$$

if $\left\|y^{-1} \circ x\right\|<M\left\|x^{-1} \circ z\right\|$, then

$$
\left\|y^{-1} \circ x\right\|<M c\left\|z^{-1} \circ x\right\|
$$

and

$$
\begin{aligned}
\left\|y^{-1} \circ z\right\| & \leq c\left(\left\|y^{-1} \circ x\right\|+\left\|x^{-1} \circ z\right\|\right)<c\left(M\left\|x^{-1} \circ z\right\|+\left\|x^{-1} \circ z\right\|\right) \\
& =c(1+M)\left\|x^{-1} \circ z\right\| .
\end{aligned}
$$


It implies

$$
\begin{aligned}
\left|X_{i} u(x)-X_{i} u(z)\right| \leq & \int_{\left\|y^{-1} \circ x\right\| \geq \frac{M}{c}\left\|z^{-1} \circ x\right\|} \frac{c\left\|x^{-1} \circ z\right\|}{\left\|y^{-1} \circ x\right\|^{Q}}|L u(y)| d y \\
& +\int_{\left\|y^{-1} \circ x\right\|<M c\left\|z^{-1} \circ x\right\|} \frac{c}{\left\|y^{-1} \circ x\right\|^{Q-1}}|L u(y)| d y \\
& +\int_{\left\|y^{-1} \circ z\right\|<c(1+M)\left\|x^{-1} \circ z\right\|} \frac{c}{\left\|y^{-1} \circ z\right\|^{Q-1}}|L u(y)| d y \\
\doteq & I_{4}+I_{5}+I_{6} .
\end{aligned}
$$

Applying Lemma 4 ( $\alpha=0$ and $\sigma=\frac{M}{c}$ ) and $\lambda<Q$, there exists a constant $c=$ $c(p, \lambda, \sigma)>0$ such that

$$
I_{4} \leq c\|L u\|_{L^{p, \lambda}(G)}\left\|z^{-1} \circ x\right\|\left\|z^{-1} \circ x\right\|^{\frac{\lambda-Q}{p}}=c\|L u\|_{L^{p, \lambda}(G)}\left\|z^{-1} \circ x\right\|^{\frac{p+\lambda-Q}{p}} ;
$$

from Lemma 4 ( $\beta=1$ and $\sigma=M c ; \beta=1$ and $\sigma=c(1+M)$, respectively) and $\lambda+p>Q$, it gets

$$
I_{5} \leq c\|L u\|_{L^{p, \lambda}(G)}\left\|z^{-1} \circ x\right\|^{\frac{p+\lambda-Q}{p}}
$$

and

$$
I_{6} \leq c\|L u\|_{L^{p, \lambda}(G)}\left\|z^{-1} \circ x\right\|^{\frac{p+\lambda-Q}{p}} .
$$

In conclusion we reach to (1.4).

\section{REFERENCES}

[1] E. Barucci, S. Polidoro, and V. Vespri, "Some results on partial differential equations and Asian options," Math. Models Methods Appl. Sci., vol. 11, no. 3, pp. 475-497, 2001.

[2] M. Bramanti and L. Brandolini, " $L^{p}$ estimates for uniformly hypoelliptic operators with discontinuous coefficients on homogeneous groups," Rend. Sem. Mat. Univ. Pol. Torino, vol. 58, pp. 389-433, 2000.

[3] S. Chandrasekhar, "Stochastic problems in physics and astronomy," Rev. Mod. Phys., vol. 15, pp. 1-89, 1943.

[4] S. Chapman and T. G. Cowling, The mathematical theory of nonuniform gases, 3rd ed. Cambridge: Cambridge University Press, 1990.

[5] J. J. Duderstadt and W. R. Martin, Transport theory, ser. A Wiley-Interscience Publication. New York: John Wiley \& Sons, 1979.

[6] G. B. Folland, "Subelliptic estimates and function spaces on nilpotent Lie groups," Ark. Mat., vol. 13, pp. 161-207, 1975.

[7] N. Garofalo and E. Lanconelli, "Level sets of the fundamental solution and Harnack inequality for degenerate equations of Kolmogorov type," Trans. Am. Math. Soc., vol. 321, no. 2, pp. 775-792, 1990.

[8] L. Hörmander, "Hypoelliptic second order differential equations," Acta Math., vol. 119, pp. 147$171,1967$.

[9] L. P. Kuptsov, "Fundamental solutions for a class of second-order elliptic-parabolic equations," English Transl. Differential Equations, vol. 8, pp. 1269-1278, 1972. 
[10] L. P. Kuptsov, "Mean value theorem and a maximum principle for Kolmogorov's equation," English Transl. Math. Notes, vol. 15, pp. 280-286, 1974.

[11] E. Lanconelli and S. Polidoro, "On a class of hypoelliptic evolution operators," Rend. Sem. Mat. Univ. Pol. Torino, vol. 52, pp. 29-63, 1994.

[12] A. Pascucci, "Hölder regularity for a Kolmogorov equation," Trans. Am. Math. Soc., vol. 355, no. 3, pp. 901-924, 2003.

[13] A. Pascucci and S. Polidoro, "On the Harnack inequality for a class of hypoelliptic evolution equations,” Trans. Am. Math. Soc., vol. 356, no. 11, pp. 4383-4394, 2004.

[14] S. Polidoro and M. A. Ragusa, "Sobolev-Morrey spaces related to an ultraparabolic equation," Manuscr. Math., vol. 96, no. 3, pp. 371-392, 1998

\section{Authors' addresses}

\section{Yuexia Hou}

Northwestern Polytechnical University, Department of Applied Mathematics; Key Laboratory of Space Applied Physics and Chemistry, Ministry of Education, Xi' an, Shaanxi, 710129, China

E-mail address: houyuexia@126.com

\section{Xiaojing Feng}

Northwestern Polytechnical University, Department of Applied Mathematics; Key Laboratory of Space Applied Physics and Chemistry, Ministry of Education, Xi' an, Shaanxi, 710129, China

E-mail address: fxj467@mail.nwpu.edu.cn

\section{Xuewei Cui}

Northwestern Polytechnical University, Department of Applied Mathematics; Key Laboratory of Space Applied Physics and Chemistry, Ministry of Education, Xi'an, Shaanxi, 710129, China

E-mail address: c88xw@163.com 